\title{
Second guesses and the attention-switching model for successiveness discrimination
}

\author{
LORRAINE G. ALLAN \\ McMaster University, Hamilton, Ontario, Canada
}

\begin{abstract}
The present note demonstrates that Baron's (1971) second-guess data are not inconsistent with the basic assumptions of Kristofferson's attention-switching model.
\end{abstract}

Baron (1971) has presented data which he has interpreted as providing strong evidence against the account of successiveness discrimination provided by a class of models or theories known as discrete psychological moment theory. One of the models that Baron classifies as a moment theory is the attention-switching model developed by Kristofferson $(1967 \mathrm{a}, 1967 \mathrm{~b}, 1970)$ and elaborated by Allan and Kristofferson (1974). The purpose of the present note is to demonstrate that, contrary to Baron's conclusions, his data are not inconsistent with the basic assumptions of the attention-switching model.

The defining characteristic of a discrete moment theory is that internal or psychological time is divided into discrete, nonoverlapping units called moments. Moment theory specifies that if two stimuli that are separated in external time fall in the same moment, all order information is lost. The two externally separated stimuli will be indiscriminable from the simultaneous presentation of the two stimuli, as far as order of occurrence is concerned. If the two externally separated stimuli fall in two different moments, their order will always be correctly perceived. The probability of two stimuli falling in two different moments will depend on the size of the moment, and on the temporal separation between the two stimuli.

In one of Baron's experiments (Experiment 1), three visual stimuli were presented on each trial. One stimulus preceded, by 8 to $48 \mathrm{msec}$, the other two, which were simultaneous. The observer's task was to identify the stimulus that came first. After making his identification, he was required to make a second response, which had to be different from his first response, concerning which of the three stimuli was presented first. In other words, he had to rank the three stimuli as to their degree of "firstness." No trial-by-trial feedback was given. If it is assumed that the observer always makes a correct first response when order information is available, then moment theory specifies that second responses following incorrect first responses cannot be based on order

The preparation of the paper was supported by Grant A8260 from the National Research Council of Canada. A more detailed version is available as McMaster Technical Report No. 57, 1974. I would like to express my thanks to Jonathon Baron for his helpful comments on an earlier draft of this paper. information. Since an incorrect first response is always the result of all three stimuli falling in the same moment, a correct second response is just a correct "guess." Using this argument as a basis, Baron states that moment theories predict that the probability of a correct second guess following an incorrect first response has to be independent of the temporal separation between the stimuli, and will always be at the chance level of $50 \%$ correct.

For three of the five observers in Baron's experiment, the probability of a correct second guess following an incorrect first response was an increasing function of the temporal separation between the stimuli, for one observer it was a slightly decreasing function, and for the remaining observer it was fairly constant at $50 \%$. The data from four of the observers are inconsistent with the $50 \%$ prediction of moment theories.

In the present note it will be shown that even though the attention-switching model does specify that second guesses following incorrect first responses are not based on order information, the probability of correct second guess following an incorrect first response can be a function of the temporal separation between the stimuli, and can vary from 0.00 to 1.00 . Baron's data, therefore, are not inconsistent with the basic assumptions of the attention-switching model.

\section{Second Guesses and the Attention-Switching Model}

The attention-switching model postulates that the processing of temporal order information is under the control of an internal timing mechanism which generates a succession of equally spaced points in time. These points occur at the rate of one every $\mathrm{q} \mathrm{msec}$, and their occurrence is independent of the presentation of an external stimulus. It is assumed that there are independent input channels, that only one channel can be attended to at a time, and that attention can be switched from one channel to another only at an internally geenrated time point. Thus, for the temporal order of two stimuli in independent input channels to be correctly discriminated, it is necessary to observe the occurrence of one, switch attention to the channel of the second, and then observe the occurrence of the second. If the second stimulus has already occurred by the time the switching operation is completed, then the two stimuli 
cannot be distinguished from simultaneous stimuli and there is no order information. Thus, for the perception of temporal order, a time point must fall in the interval between two stimuli. The source of errors is the waiting time before attention can switch from one channel to another. The waiting time has a uniform distribution over a range from 0 to $\mathrm{q}$ msec.

A pair of stimuli may fall anywhere on the time continuum with respect to the internal time points. Assuming that the stimuli have equal transmission times, the probability that a time point will fall between two stimuli separated by $\mathrm{t} \mathrm{msec}$ is $a_{\mathrm{t}}=\mathrm{t} / \mathrm{q}$ fọr $0 \leqslant t \leqslant q$.

In Baron's experiment, there are three stimuli, $S_{X}$, $S_{Y}$, and $S_{Z}$, and three equally likely stimulus patterns: $S_{X}$ followed $t$ msec later by $S_{Y}$ and $S_{Z}$, a $S_{X}-S_{Y} S_{Z}$ pattern; $S_{Y}$ followed $t$ msec later by $S_{X}$ and $S_{Z}$, a $S_{Y}-S_{X} S_{Z}$ pattern; $S_{Z}$ followed $t$ msec later by $S_{X}$ and $S_{Y}$, a $S_{Z}-S_{X} S_{Y}$ pattern. In Figure 1, a possible version of the attention-switching model for the Baron experiment is presented schematically for $0 \leqslant \mathrm{t} \leqslant \mathrm{q}$. The parameter $\mathrm{P}_{\mathrm{i}}$ represents the probability of attending to channel $i$ when the first stimulus is presented, for $\mathrm{i}$ equal to $\mathrm{X}, \mathrm{Y}$, or $\mathrm{Z}$, and $\Sigma P_{i}=1.0$. If the observer is attending to the channel of the stimulus that is presented first, then with probability $a_{t}$ he will perceive that stimulus as occurring before the other two, an information state, and with probability $\left(1-a_{t}\right)$ he will have no order information, a noninformation state. If he is not attending to the channel of the stimulus that is presented first, he will always enter a noninformation state. When in an information state, he will make a correct response with probability 1.0. When in a noninformation state, he will guess. He will respond that $S_{i}$ occurred first, an $R_{i 1}$ response, with probability $\lambda_{i}$, where $\Sigma \lambda_{i}=1.0$. In order to reduce the number of parameters, it assumed that the observer's response tendency, when in a noninformation state, does not depend upon the channel attended to. His second response, $\mathrm{R}_{\mathbf{j} 2}$, is required (by the experimenter) to be different from his first and therefore will never be based on order information. Thus, the second response is always a guess, and it is assumed that the probability of a $R_{j} 2$ response, $K_{i j}$, for $j$ equal to $X, Y$, or $Z$, and $j \neq i$, is dependent upon the first response made.

Baron argued that the probability of a correct second response given an incorrect first response, $\mathrm{P}\left(\mathrm{C}_{2} \mid \overline{\mathrm{C}}_{1}\right)$, should be 0.5 in his experimental task for all values of $t$. According to the attention-switching model, as presented in Figure 1,

$P\left(C_{2} \mid \bar{C}_{1}\right)$

$=1-\alpha_{4}\left[\lambda_{X} K_{X Y}\left(P_{Y}-P_{Z}\right)+\lambda_{Y} K_{Y X}\left(P_{X}-P_{Z}\right)+\lambda_{Z} K_{Z X}\left(P_{X}-P_{Y}\right)+\lambda_{Z} P_{Y}+\left(\lambda_{X}+\lambda_{Y}\right) P_{Z}\right]$ $2-\alpha_{t}\left[1-\lambda_{X} P_{X}-\lambda_{Y} P_{Y}-\lambda_{Z} P_{Z}\right]$
Let us consider some specific examples of possible attention preferences and response biases an observer might adopt in Baron's task. Suppose he decides to always attend to the same channel, for example channel $X$, at the beginning of a trial. For $P_{X}=1$, he will enter an information state only on $\mathrm{S}_{X}-\mathrm{S}_{Y} \mathrm{~S}_{Z}$ trials, and therefore when order information is available he will always respond $\mathrm{RX}_{\mathrm{X}}$. When in a noninformation state, he has a very strong bias for saying $S_{Y}$ occurred first as his first response $\left(\lambda_{Y}=\right.$ 1), and $S_{Z}$ occurred first as his second response $(\mathrm{KYZ}$ $=1$ ). Substituting $\mathrm{PX}_{X}=1, \lambda Y=1$, and $\mathrm{KYZ}=1$ in Equation 1 yields

$$
\mathrm{P}\left(\mathrm{C}_{2} \mid \overline{\mathrm{C}}_{1}\right)=\frac{1}{2-\alpha_{\mathrm{t}}} .
$$

Consider first the case for $a_{\mathrm{t}}=1$. The observer will always make a correct first response on $\mathrm{S}_{X}-\mathrm{S}_{Y} \mathrm{~S}_{Z}$ trials because he will always be able to switch from clannel $X$ to one of the other channels before the stimulus in the switcned-to channel is presented. The observer will also always make a correct first response on $\mathrm{S}_{Y}-\mathrm{S}_{X} \mathrm{~S}_{Z}$ trials because of his strong bias to respond $R_{Y 1}$. Thus, he will only make an incorrect response on one-third of the trials, on $S_{Z}-S_{X} S_{Y}$ trials. On these incorrect first response trials, he will always be correct on his second response because of his strong bias to response $R_{Z 2}$. Thus, for $a_{t}=1$, the probability of correct second response, given an incorrect first response, is 1.0. Examination of Equation 2 shows that as $a_{t}$ decreases to 0.0 , $\mathrm{P}\left(\mathrm{C}_{2} \mid \overline{\mathrm{C}}_{1}\right)$ decreases to 0.5 .

Another strategy the observer might adopt leads to the following combination of parameters; $P X=1, \lambda Y$ $=1$, and $\mathrm{KYX}=1$. When the observer has no order information, he responds $\mathrm{R}_{\mathrm{Y} 1}$ first and $\mathrm{R}_{\mathrm{X} 2}$ second. Substituting in Equation 1,

$$
P\left(C_{2} \mid \vec{C}_{1}\right)=\frac{1-\alpha_{t}}{2-\alpha_{t}}
$$

Here $\mathrm{P}\left(\mathrm{C}_{2} \mid \overline{\mathrm{C}}_{1}\right)$ is an inverse function of $a_{t}$. As $a_{t}$ increases from 0.0 to $1.0, \mathrm{P}\left(\mathrm{C}_{2} \mid \mathrm{C}_{1}\right)$ increases from 0.5 to 0.0 .

The above two examples of parameter combinations represent extreme strategies for an observer to adopt. However, it is true that as long as the observer is somewhat biased (a) in his channel preference or (b) in his responses when he is in a noninformation state, $P\left(C_{2} \mid \bar{C}_{1}\right)$ will not necessarily equal 0.50 . Thus, the finding by Baron that second responses are not at chance when first responses are incorrect, does not by itself contradict the basic assumptions of the attention-switching model. According to the attention-switching model, even though second responses following incorrect first responses are not determined by order information, the probability of a correct second response is not necessarily 0.5 . 


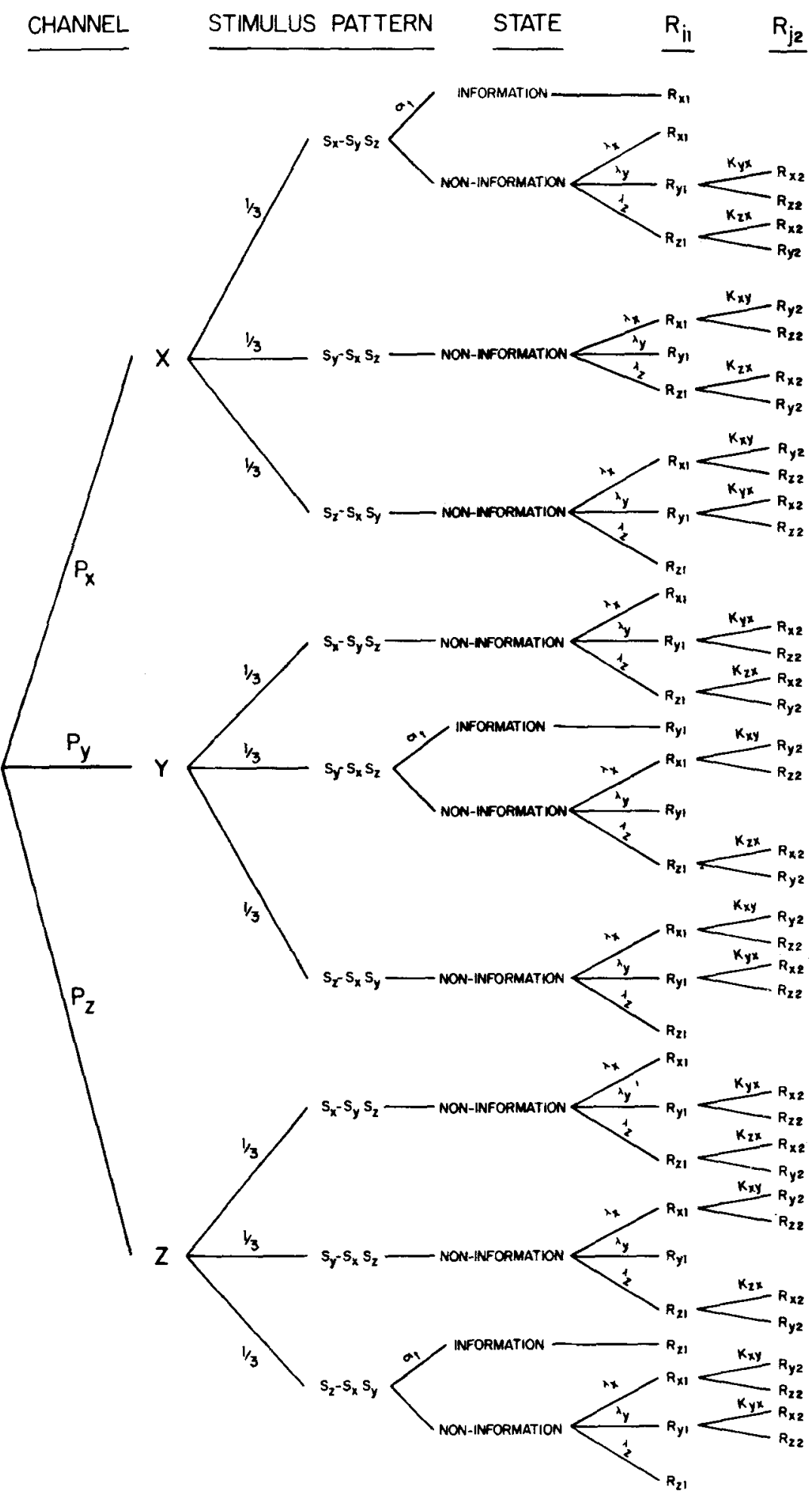

Figure 1. Schematic of a possible version of the attention-switching model for Baron's second guess task. Since Equation 1 is concerned only with second responses following incorreet first responses, the branches diagraming the relationships between second responses following correct first response are omitted. 
While it is true that the existence of channel preferences and response biases may result in $\mathrm{P}\left(\mathrm{C}_{2} \mid \overline{\mathrm{C}}_{1}\right) \neq 0.5$ and be dependent upon $\mathrm{t}$, it is also the case that some combination of preferences and biases result in $\mathrm{P}\left(\mathrm{C}_{2} \mid \overline{\mathrm{C}}_{1}\right)=0.5$ for all values of $t$. For example, substituting $\mathrm{P}_{\mathrm{X}}=1, \lambda_{\mathrm{Y}}=.50, \lambda_{\mathrm{Z}}=.40$, $\mathrm{K}_{\mathrm{YX}}=.26$, and $\mathrm{K}_{\mathrm{ZX}}=.80$ in Equation 1 yields

$$
\mathrm{P}\left(\mathrm{C}_{2} \mid \overline{\mathrm{C}}_{1}\right)=\frac{1-.45 \alpha_{\mathrm{t}}}{2-.90 \alpha_{\mathrm{t}}}=\frac{1}{2} \text {. }
$$

In sum, if the observer has attention preferences or response biases, we could find that $P\left(C_{2} \mid \bar{C}_{1}\right)$ varies directly with $t$, as was the case with three of Baron's observers; that $\mathrm{P}\left(\mathrm{C}_{2} \mid \overline{\mathrm{C}}_{1}\right)$ varies inversely with $\mathrm{t}$, as was the case with one observer; or that $P\left(C_{2} \mid \bar{C}_{1}\right)=$ 0.5 and is independent of $t$, as was the case with one observer. The important factor appears to be the particular combination of the various parameters.

\section{Second Guesses and Classical Moment Theory}

While Baron's conclusion that $\mathrm{P}\left(\mathrm{C}_{2} \mid \overline{\mathrm{C}}_{1}\right)$ must equal 0.5 is not true for the attention-switching model, it is true for discrete moment theory as discussed by Stroud (1955) and Allport (1968). Moment theory can be characterized in the following way. Internal or psychological time is quantized into discrete nonoverlapping units called moments. If stimuli fall in the same moment, all order information is lost, and the observer's response is not determined by order information. If stimuli fall in different moments, order information is perfect. In Figure 2, this version of moment theory is presented schematically. If it is assumed that the transmission times for the three stimuli are the same, then with probability $a_{t}$ the observer enters an information state, and with probability 1.0 he makes a correct first response. With probability $\left(1-a_{t}\right)$, he enters a noninformation state, and with probability $\lambda_{\mathrm{il}}$, he response $\mathrm{R}_{\mathrm{il}}$. Again, his second response, $\mathbf{R}_{\mathbf{j} 2}$, is dependent upon his first response.

In comparing the attention-switching model with moment theory, the important distinction is that in moment theory the probability of obtaining order information about a particular stimulus pattern depends upon the value of $t$ and the size of the moment. In the attention-switching model, the probability of obtaining order information about a particular stimulus pattern also depends upon the channel the observer is attending to.

From Figure 2 it can be seen that moment theory predicts

$$
\mathrm{P}\left(\mathrm{C}_{2} \mid \overline{\mathrm{C}}_{1}\right)=0.5
$$

for all values of $a_{t}$. Thus, Baron's second guess data do reject classical moment theory, if it is assumed that

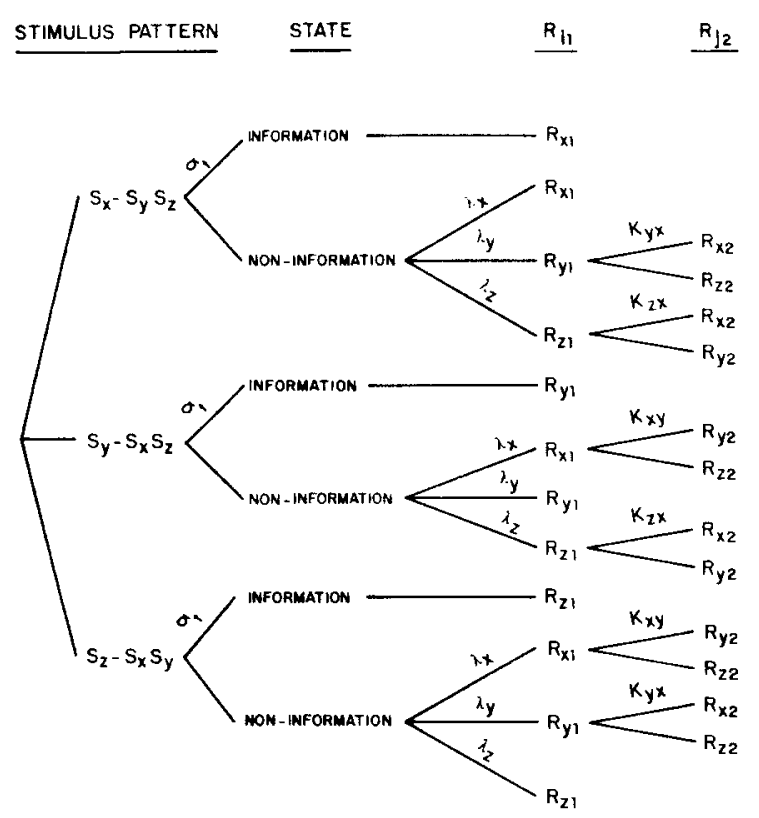

Figure 2. Schematic of moment theory.

the observer always makes a correct response when the order information is available to him, and that the transmission times of the three stimuli are the same.

\section{Concluding Comments}

The present note argues that the attentionswitching model should not be classified as a discrete moment model. Although Baron's (1971) conclusion about second guesses is correct for moment theory, it is not correct for the attention-switching model. Baron's second guess data to not contradict the basic assumptions of the attention-switching model.

\section{REFERENCES}

Allan, L. G., \& Kristofferson, A. B. Successiveness discrimination: Two models. Perception \& Psychophysics, 1974, 15, 37-46.

Allport, D. A. Phenomenal simultaneity and the perceptual moment hypothesis. British Journal of Psychology, 1968, 59, $395-406$.

Baron, J. The threshold for successiveness. Perception \& Psychophysics, 1971, 10, 201-207.

Kristofferson, A. B. Attention and psychophysical time. Acta Psychologica, 1967, 27, 92-100. (a)

Kristofferson, A. B. Successiveness discrimination as a two state quantal process. Science, 1967, 158, 1337-1339. (b)

Kristofferson, A. B. Attention. In R. M. Patton, T. A. Tanner, J. Markowitz, and J. A. Swets (Eds.), Applications of research on human decision making. Washington, D.C: NASA, 1970. Pp. 49-64.

Stroud, J. M. The fine structure of psychological time. In H. Quastler (Ed.), Information theory in psychology. New York: Free Press, 1955.

(Received for publication June 24, 1974; revision received August 14, 1974.) 